\title{
Unravelling the Surface Chemistry of Metal Oxide Nanocrystals, the Role of Acids and Bases
}

Jonathan De Roo ${ }^{1,2}$, Freya Van den Broeck ${ }^{4}$, Katrien De Keukeleere ${ }^{1}$, José C. Martins ${ }^{4}$, Isabel Van Driessche $^{1}$, Zeger Hens ${ }^{2,3}$

${ }^{1}$ Sol-gel Centre for Research on Inorganic Powders and Thin films Synthesis (SCRiPTS), Ghent University, Belgium

${ }^{2}$ Physics and Chemistry of Nanostructures (PCN), Ghent University, Belgium

${ }^{3}$ Center for nano and biophotonics (NB-Photonics), Ghent University, Belgium

${ }^{4} \mathrm{NMR}$ and structural analysis unit, Ghent University, Belgium

Address (Jonathan De Roo, Katrien De Keukeleere, Isabel Van Driessche, Zeger Hens): Krijgslaan 281 S3, 9000 Gent, Belgium

Address (Freya Van den Broeck, José C. Martins): Krijgslaan 281 S4, 9000 Gent, Belgium

Corresponding author:

Zeger Hens

zeger.hens@ugent.be

Tel: 003292644863

Fax: 003292644983

Key words: hafnium oxide, nonaqueous, ligand exchange, benzyl alcohol

\section{Abstract}

We synthesized $\mathrm{HfO}_{2}$ nanocrystals from $\mathrm{HfCl}_{4}$ using a surfactant-free solvothermal process in benzylalcohol and found that the resulting nanocrystals could be transferred to nonpolar media using a mixture of carboxylic acids and amines. Using solution ${ }^{1} H$ NMR, FTIR and elemental analysis, we studied the details of the transfer reaction and the surface chemistry of the resulting sterically stabilized nanocrystals. As synthesized nanocrystals are charge-stabilized by protons, with chloride acting as the counterion. Treatment with only carboxylic acids does not lead to any binding of ligands to the $\mathrm{HfO}_{2}$ surface. On the other hand, we find that the addition of amines provides the basic environment in which carboxylic acids can dissociate and replace chloride. This results in stable, aggregate-free dispersions of $\mathrm{HfO}_{2}$ nanocrystals, sterically stabilized by carboxylate ligands. Moreover, titrations with deuterated carboxylic acid show that the charge on the carboxylate ligands is balanced by co-adsorbed protons. Hence, opposite from the X-type/non-stoichiometric nanocrystals picture prevailing in literature, one should look at $\mathrm{HfO}_{2} /$ carboxylate nanocrystals as systems where carboxylic acids are dissociatively adsorbed to bind to the nanocrystals. Similar results were obtained with $\mathrm{ZrO}_{2}$ NCs. Since proton accommodation on the surface is most likely due to the high 
Brønsted basicity of oxygen, our model could be a more general picture for the surface chemistry of metal oxide nanocrystals with important consequences on the chemistry of ligand exchange reactions.

\section{Introduction}

Metal oxide nanocrystals (MONCs) are an important class of nanomaterials regarding their potential in medicine, ${ }^{1}$ (photo)catalysis, ${ }^{2,3}$ gas sensing, ${ }^{4}$ magnetic applications, ${ }^{5}$ solar cells, ${ }^{6}$ transparent electrodes, ${ }^{7}$ lithium ion batteries ${ }^{8,9}$ and light emitting diodes. ${ }^{10}$ Their suitability for a specific application depends on their properties, such as chemical nature, size, shape, size dispersion, crystallinity, colloidal stability and surface composition, and their eventual industrial implementation requires fast and robust syntheses with a high yield, cheap precursors and low energy input. Many research activities have therefore been dedicated to the development of various synthetic strategies, where solution-based approaches stand out since they can be applied to prepare a wide range of MONCs with an often exceptional control over size, size-dispersion and shape.

Using solution-based approaches in aqueous media, it proved possible to synthesize monodisperse colloids in short reaction times, but the particles were often very large $(>100 \mathrm{~nm})$ and amorphous. ${ }^{11,12}$ The subsequent crystallization step induced undesired agglomeration. In addition, the final properties of the nanoparticles were found to be very sensitive to the precise reaction conditions (e.g., $\mathrm{pH}$, temperature, etc.). In surfactant-assisted nonaqueous methods - typically carried out by hot injection or heating up - large quantities of ligands are used to control nucleation, (anisotropic) growth and colloidal stability, often leading to very monodisperse MONCs. ${ }^{5,13-16}$ In general, these syntheses proceed at high temperatures $\left(>300^{\circ} \mathrm{C}\right)$ to quickly decompose the metal precursor and obtain crystalline products. Successful syntheses require a rigorous control of heating rate, precursor addition rate, etc., whereas further complications may result from structural changes of the surfactants at the high temperatures used ${ }^{17}$ or from impurities in surfactants, which can be more decisive to the outcome of a synthesis than the surfactant itself. ${ }^{18,19}$ Contrarily, surfactant-free nonaqueous methods are based on chemically robust procedures with high yields. ${ }^{20}$ The metal precursors - typically cheap metal salts - are simply mixed with a nontoxic solvent such as benzyl alcohol or benzyl amine, and heated for several days in an autoclave. The reduced hydrolysis and condensation rates result in crystalline particles even at moderate temperatures of about $200{ }^{\circ} \mathrm{C}$. The slow autoclave process is objectionable but it was shown that these reactions can be accelerated considerably by using microwave heating, while still retrieving products with excellent crystallinity. ${ }^{21,22}$ With this approach, a vast range of (binary or doped) MONCs can be prepared but it offers less control over size and shape of the NCs. Moreover, the resulting MONCs suffer from agglomeration and lack of redispersibility. ${ }^{20}$ 
A possibly generic solution to form stable, aggregate-free dispersions of MONCs generated by a surfactant-free synthesis could be a post-synthetic surface modification in nonpolar solvents where a small amount of pristine ligands is introduced, providing stabilization by steric hindrance. In addition, traces of unwanted side products can be removed concomitantly. In some respects, this concept is the reverse of transferring colloidal nanocrystals (NCs) - synthesized using hot injection - to polar solvents via ligand exchange $\mathrm{e}^{23,24}$ and a few examples can already be found in the literature. Aggregate-free suspensions of $\mathrm{ZrO}_{2}{ }^{25,26}$ and $\mathrm{Fe}_{3} \mathrm{O}_{4}{ }^{27} \mathrm{NCs}$ - synthesized via surfactantfree methods - were obtained with fatty acids, which was ascribed to their strong and selective interaction with the MONC surface. On the other hand, the surface modification of ITO ${ }^{25}$ (indium tin oxide) proved successful with long-chain primary amines, albeit due to a weak interaction and in the case of $\mathrm{HfO}_{2} \mathrm{NCs}$, only a combination of fatty acid and alkylamine led to a stable dispersion of individual NCs. ${ }^{21}$ Although all examples illustrate the benefit of the approach by the minimal amount of surfactant required to obtain a stable, aggregate-free dispersion of MONCs, the diversity and apparent randomness of the recipes indicate that a rational basis for the surface functionalization of MONCs is lacking. In this respect, an in-depth understanding of the surface chemistry during all stages of synthesis and functionalization would allow for more judicious surface modification schemes.

In this paper, we take the observation that two types of surfactants are needed to obtain stable, aggregate-free dispersions of $\mathrm{HfO}_{2}$ or $\mathrm{ZrO}_{2} \mathrm{NCs}-$ synthesized using the respective metal chloride and benzylalcohol - as a starting point to study the surface chemistry of these MONCs. Assynthesized $\mathrm{HfO}_{2}$ or $\mathrm{ZrO}_{2} \mathrm{NCs}$ are charge stabilized in polar media by an acid/base equilibrium ${ }^{21}$ and as we show here, have hydrogen chloride adsorbed at their surface in nonpolar media. Focusing on $\mathrm{HfO}_{2} \mathrm{NCs}$, we demonstrate with solution ${ }^{1} \mathrm{H}$ NMR and infrared spectroscopy that exposure of the NCs to a mixture of carboxylic acids and alkylamines results in $\mathrm{HfO}_{2} \mathrm{NCs}$ capped by carboxylate ligands whereas alkylamines are lost during successive purification steps. The bound ligands exhibit a self-exchange upon addition of excess carboxylic acid similar to CdSe NCs synthesized in the presence of carboxylic acids using hot injection. ${ }^{28}$ However, unlike CdSe or PbS $\mathrm{NCs},{ }^{28,29}$ we find that $\mathrm{HfO}_{2} \mathrm{NCs}$ also accommodate protons on their surface, where the amount of protons matches the amount of carboxylate moieties. The fact that the MONCs are stabilized by dissociated Brønsted acids, i.e., a proton and the conjugated base, and not by the conjugated base only is most likely linked to the higher Brønsted basicity of oxygen as compared to the heavier chalcogens. During the polar-apolar phase transfer, the long-chain amine is also found to act as a Brønsted base, enabling the replacement of the initially present strong acid (hydrogen chloride) by the weaker carboxylic acids. We thus conclude that MONCs can exhibit a surprisingly rich surface 
chemistry, where the presence of acid/base equilibria extends the prevailing picture of metal sulfide, selenide or phosphide NCs synthesized by hot injection. ${ }^{30,31}$

\section{Experimental methods}

Nanocrystal synthesis. $\mathrm{HfO}_{2} \mathrm{NCs}$ were synthesized via an established microwave-assisted solvothermal process with some slight modifications. ${ }^{21}$ The precursor preparation was executed in a nitrogen filled glovebox. Under vigorous stirring, $0.5 \mathrm{~mL}$ of dibenzyl ether was added to $0.4 \mathrm{mmol}$ of hafnium chloride in a $10 \mathrm{~mL}$ microwave vial. Then quickly $4 \mathrm{~mL}$ of benzyl alcohol is added, yielding a clear and colorless solution after 5 minutes of stirring. The solution was subjected to microwave heating with the following temperature settings: 5 minutes at $60{ }^{\circ} \mathrm{C}$ and 3 hours at 220 ${ }^{\circ} \mathrm{C}$. After synthesis the phase separated mixture is transferred to a plastic centrifugation tube and 3 $\mathrm{mL}$ of diethyl ether is added. After mild centrifugation (2000 rpm, 2min) two clear and transparent phases are observed. The organic (top) phase is removed and ethanol is added to the aqueous (bottom) phase yielding $2 \mathrm{~mL}$ of a clear suspension. The particles are precipitated and washed once with diethyl ether. Finally the particles are redispersed in chloroform and typically $0.2 \mathrm{mmol}$ of fatty acid (dodecanoic acid, oleic acid or 10-undecenoic acid) is added to the milky suspension. Under stirring, amine (oleylamine or triethylamine) was added until a colorless and transparent suspension is obtained. As a standard protocol, $0.15 \mathrm{mmol}$ (i.e., $50 \mu \mathrm{L}$ ) is used since this already results in optically clear dispersions. The particles can be purified by adding a non-solvent (acetonitrile, acetone), followed by centrifugation and resuspension in chloroform. $\mathrm{ZrO}_{2} \mathrm{NCs}_{\text {were }}$ synthesized via the same protocol but with a different precursor quantity $(0.56 \mathrm{mmol}$ zirconium chloride) and reaction time ( 4 hours at $220^{\circ} \mathrm{C}$ ). More comments on the synthesis procedure can be found in the SI.

Characterization: For Dynamic Light Scattering (DLS) and zeta potential measurements a Malvern Nano ZS was used in backscattering mode $\left(173^{\circ}\right)$. Semi-quantitative analysis was obtained by calibration of a Rigaku CG Energy Dispersive X-ray Fluorescence (EDXRF) analyzer working with the FP quantitative analysis (RPF-SQX). Photometric determination of chloride was performed with the VWR chloride test nr. 1.14897.0001. Transmission electron microscopy (TEM) images were taken on a JEOL JEM-2200FS TEM with Cs corrector. For X-ray diffraction (XRD) characterization a Thermo Scientific ARL X'tra X-ray diffractometer was used with the CuKa line as the primary source. For infrared measurements a Perkin Elmer FT-IR spectrometer spectrum 1000, equiped with a HATR module was used.

Solution ${ }^{1} \mathbf{H}$ NMR Characterization: Nuclear Magnetic Resonance (NMR) measurements were recorded on a Bruker Avance III Spectrometer operating at a ${ }^{1} \mathrm{H}$ frequency of $500.13 \mathrm{MHz}$ and 
equipped with a BBI-Z probe. For each NMR measurement a $750 \mu \mathrm{L}$ ampule of dry deuterated solvent was used. The sample temperature was set to $298.2 \mathrm{~K}$. One dimensional (1D) ${ }^{1} \mathrm{H}$ and 2D NOESY (Nuclear Overhauser Effect Spectroscopy) spectra were acquired using standard pulse sequences from the Bruker library. For the quantitative 1D ${ }^{1} \mathrm{H}$ measurements, 64k data points were sampled with the spectral width set to $16 \mathrm{ppm}$ and a relaxation delay of $30 \mathrm{sec}$. NOESY mixing time was set to $300 \mathrm{~ms}$ and 2048 data points in the direct dimension for 512 data points in the indirect dimension were typically sampled, with the spectral width set to $11.5 \mathrm{ppm}$. Off-resonance ROESY (Rotating-frame Nuclear Overhauser effect Spectroscopy) was implemented as described by Desvaux and Goldman. ${ }^{32}$ ROESY mixing time was set to $150 \mathrm{~ms}$ and $4 \mathrm{k}$ data points in the direct dimension for 512 data points in the indirect dimension were typically sampled, with the spectral width set to $13 \mathrm{ppm}$. Diffusion measurements (2D DOSY) were performed using a double stimulated echo sequence for convection compensation and with monopolar gradient pulses. ${ }^{33}$ Smoothed rectangle gradient pulse shapes were used throughout. The gradient strength was varied linearly from $2-95 \%$ of the probe's maximum value (calibrated at $50.2 \mathrm{G} / \mathrm{cm}$ ) in 32 or 64 steps, with the gradient pulse duration and diffusion delay optimized to ensure a final attenuation of the signal in the final increment of less than $10 \%$ relative to the first increment. For 2D processing, the spectra were zero filled until a 4096×2048 real data matrix. Before Fourier transformation, the 2D spectra were multiplied with a squared cosine bell function in both dimensions, the 1D spectra were multiplied with an exponential window function. Concentrations were obtained using the Digital ERETIC method. The diffusion coefficients were obtained by fitting the appropriate StejskalTanner equation to the signal intensity decay. ${ }^{34}$

\section{Results and discussion}

3.1 From aggregates to stable dispersions. $\mathrm{HfO}_{2} \mathrm{NCs}$ were synthesized using $\mathrm{HfCl}_{4}$ and benzyl alcohol in a microwave-assisted solvothermal synthesis. ${ }^{21}$ The XRD diffractogram (Figure 1A) confirmed the monoclinic crystal structure. Immediately after synthesis, the NCs could be dispersed in water, ethanol or methanol. Figure 1B depicts DLS measurements and TEM images of $\mathrm{HfO}_{2} \mathrm{NC}$ suspensions in ethanol. The DLS size distribution is centered at $37 \mathrm{~nm}$ and comparison with TEM images ascertains that individual NCs $(d=5.0 \mathrm{~nm})$ are aggregated in the polar solution.

It was possible to precipitate and wash the hafnia NCs with diethyl ether and subsequently redisperse them in chloroform by the addition of two surfactants: oleylamine (OAm) and dodecanoic acid (DDAc). The combination of the two was essential to obtain a transparent colloidal suspension. The addition of only OAm or only DDAc resulted in dispersions turbid upon visual inspection. Moreover, in chloroform the average hydrodynamic diameter (nanocrystal and ligand 
shell) is $9 \mathrm{~nm}$ according to DLS (Figure 1B). The TEM pictures in the inset also confirm the absence of large aggregates in chloroform. In conclusion, the combination of OAm and DDAc allowed the phase transfer from polar to nonpolar media and the concomitant de-aggregation of the hafnia NCs.

By optimizing the precursor concentration and the reaction time, we were also able to obtain zirconium oxide NCs via the same synthesis protocol. In contrast to $\mathrm{ZrO}_{2} \mathrm{NCs}$ synthesized from zirconium isopropoxide and benzylalchol, ${ }^{25}$ these particles showed no affinity towards mere carboxylic acids and again the addition of OAm was indispensable. In addition, the XRD, TEM and DLS analyses showed very similar results to those of hafnium oxide (SI). We infer that the surfactant requirements are not only dominated by the nature of the metal but also by the type of metal precursor.
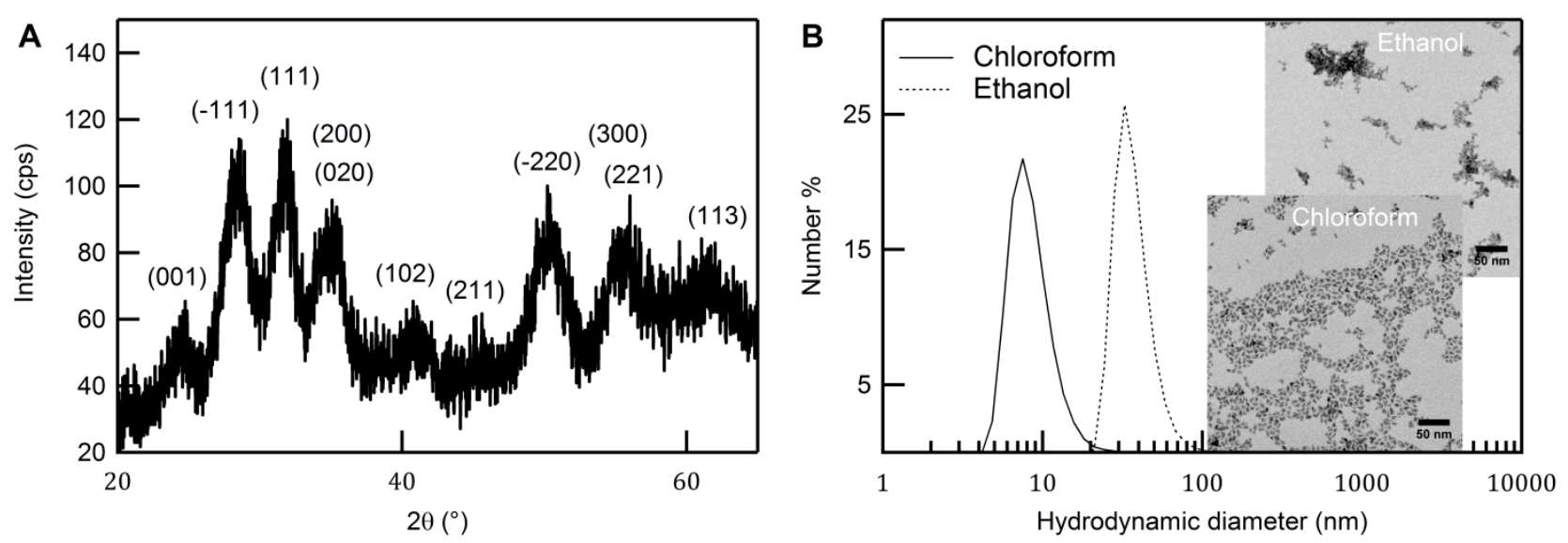

Figure 1. (A) XRD diffractogram of one batch of $\mathrm{HfO}_{2} \mathrm{NCs}$. (B) Size distribution by DLS of $\mathrm{HfO}_{2} \mathrm{NCs}$ dispersed in either ethanol (as synthesized) or chloroform (5 times purified suspension). The insets show the corresponding TEM pictures.

3.2 Characterization of the as-synthesized nanocrystal surface. The $\mathrm{HfO}_{2}$ and $\mathrm{ZrO}_{2}$ nanocrystal aggregates in ethanol feature a positive zeta potential $(+31 \mathrm{mV})$ and are therefore charge stabilized by a positive charge. In addition, XRF measurements evidenced the presence of chlorine in the suspension. From earlier work we know that the metal chloride precursor undergoes partial exchange with benzyl alcohol, resulting in the release of $\mathrm{HCl}$ and the formation of hafnium oxide via a subsequent ether elimination step. ${ }^{21}$ In addition, $\mathrm{HCl}$ catalyzes the direct conversion of benzyl alcohol to dibenzyl ether and water. In line with zeta potential measurements in aqueous solutions, ${ }^{21}$ we conclude that released protons can adsorb on the surface of the formed NCs, ensuring charging of the surface and thus colloidal stability of the NC clusters.

When the suspension is precipitated and washed once with diethyl ether prior to functionalization, charge neutrality must be preserved and an equal amount of chloride will co-precipitate to compensate for the adsorbed protons. To measure photometrically the chloride quantity, the NCs 
were resuspended in ethanol and diluted with water after the last washing step. The measurement was repeated three times to estimate the error. Finally the amount of adsorbed chloride was determined to be $50 \pm 2 \mu \mathrm{mol}$ for one batch of $\mathrm{HfO}_{2} \mathrm{NCs}$ which is equivalent to a chloride density on the nanocrystal surface of $3.7 \pm 0.2 \mathrm{~nm}^{-2}$ (procedure and calculations in SI) This value remained the same when the NCs were washed multiple times with diethyl ether. Similarly, a chloride density of $3.4 \pm 0.4 \mathrm{~nm}^{-2}$ was determined for $\mathrm{ZrO}_{2} \mathrm{NCs}$. The chloride densities are almost identical for hafnia and zirconia which amounts in both cases to about $50 \%$ of the metal (Hf or $\mathrm{Zr}$ ) surface density. Indeed, both $\mathrm{ZrO}_{2}$ and $\mathrm{HfO}_{2} \mathrm{NCs}$ have the monoclinic crystal structure and have only slightly different lattice parameters (data in SI).

3.3 Characterization of the nanocrystal surface after functionalization. After surface modification, the NCs are sterically stabilized, providing stable dispersions in nonpolar solvents. In order to elucidate the role of the two surfactants, we examined the functionalized NCs with solution ${ }^{1} \mathrm{H}$ NMR techniques. Figure 2A depicts representative ${ }^{1} \mathrm{H}$ spectra of $\mathrm{HfO}_{2} \mathrm{NCs}$ stabilized with DDAc and OAm. Sample 1 (grey spectrum) was purified only once with a mixture of acetone and acetonitrile, sample 2 (black spectrum) was purified 5 times. For purifications purposes, we used aprotic non-solvents to avoid possible exchange reactions with the ligands. ${ }^{35}$
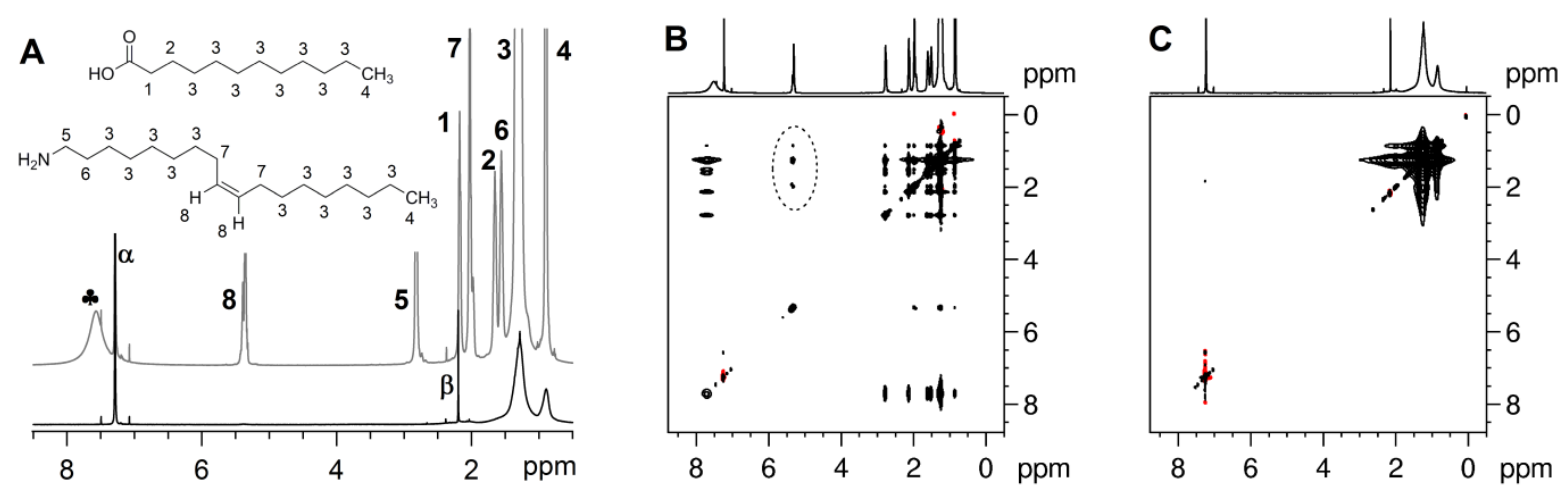

Figure 2. (A) ${ }^{1} \mathrm{H}$ NMR spectra of $\mathrm{HfO}_{2} \mathrm{NCs}$ in $\mathrm{CDCl}_{3}$ stabilized with DDAc and OAm after (grey spectrum) 1 and (black spectrum) 5 purification steps, concentration: $40 \mathrm{mg} \mathrm{HfO} 2 / \mathrm{mL}$. Greek letters refer to resonances from the solvent and the nonsolvents used during purification. Roman numbers are used to assign the protons of DDAc and OAm. The broad clover resonance is attributed to an ammonium compound (see section 3.5) (B) 2D NOESY spectrum after 1 purification step. (C) 2D NOESY spectrum after 5 purification steps.

The resonances at chemical shifts below 2 ppm are signals of aliphatic moieties that are present in both OAm and DDAc (see assignments in Figure 2A). Hence the difficulty to distinguish between both molecules in this chemical shift region. However, OAm has a characteristic resonance due to the alkene group at $5.35 \mathrm{ppm}$ so by observing that signal, information specific to OAm can be obtained. Since the alkene signal has vanished from the black spectrum (sample 2), we conclude 
that OAm was completely removed by the purification, yielding a clean spectrum with only resonances belonging to DDAc.

It is typical for bound ligands to suffer from excessive $T_{2}$ relaxation and consequently feature broadened resonances. ${ }^{36}$ The spectrum of sample 2 clearly displays this behavior - indicating bound DDAc - but the spectrum of sample $\mathbf{1}$ is more difficult to interpret. However, in order to assess ligand binding 2D NOESY NMR is more conclusive. As indicated in Figure 2B, very clear negative nOe cross peaks are visible for the distinct alkene resonance belonging to OAm, clearly indicating its proximity to the NC surface in sample 1. The nOe cross peaks between the aliphatic moieties in Figure 2C also confirm that dodecanoic acid interacts with the NCs in sample 2. Furthermore, from a DOSY experiment on sample 2, a diffusion coefficient for dodecanoic acid of $77.1 \pm 0.4 \mu \mathrm{m}^{2} / \mathrm{s}$ could be extracted. This number corresponds to a hydrodynamic diameter of $10.5 \mathrm{~nm}$, which is comparable to the DLS analysis in chloroform shown before (Figure 1B), hereby confirming that DDAc is tightly bound to the $\mathrm{HfO}_{2}$ NCs. Finally, a concentration of $22.5 \mathrm{mM}$ DDAc was determined, corresponding to a ligand density of 2.8 ligands $/ \mathrm{nm}^{2}$. This value matches reasonably well the earlier determined chloride density, a result that already hints at the interplay between both. As discussed in the Supporting Information, very similar results were observed in case of $\mathrm{ZrO}_{2}$ NCs.

3.4 Binding mode of the carboxylic acid. Given the similarity between $\mathrm{HfO}_{2}$ and $\mathrm{ZrO}_{2}$, we concentrate on the former for a detailed study of the ligand-surface interaction. Since OAm can be completely removed from the surface by repetitive purification, this concerns the binding of the carboxylic acids. For the next experiment we elected oleic acid (OAc) instead of DDAc because the alkene resonance strongly facilitates the interpretation of NMR spectra. Since both OAc and DDAc will bind to the $\mathrm{NC}$ via the carboxylic acid functionality, ${ }^{25}$ this change of ligand will not affect possible conclusions on ligand binding.

The black ${ }^{1} \mathrm{H}$ NMR spectrum in Figure $3 \mathrm{~A}$ represents a thoroughly purified sample of $\mathrm{HfO}_{2} \mathrm{NCs}$ stabilized with OAc. The sole occurrence of broadened resonances confirms that only bound OAc is retained. The grey spectrum was recorded after addition of excess OAc - one equivalent with respect to the bound OAc - to the sample. Superimposed upon the broad alkene resonance of bound OAc, a second, sharper resonance appears. Although the width of the peak suggests that this feature corresponds to free OAc, nOe cross peaks are observed for both the broadened and the sharp resonance (Figure 3B). This peculiar behavior was already observed for CdSe NCs stabilized with oleic acid and was explained by equilibria between three states of acid: bound, free and entangled in the ligand shell. ${ }^{28}$ Fast exchange between the free and the entangled state results in one single resonance with properties of both. The bound state emerges as a separate resonance due to slow exchange with the entangled state. This exchange is also directly apparent in the 2D NOESY 
spectrum as a cross peak between the two states (Figure 3B inset). Finally, a ROESY experiment confirmed that this is a chemical exchange peak and not a close-contact peak (SI). In conclusion, the same dynamic ligand-surface interaction is found in post-synthetically modified MONCs as in CdSe NCs synthesized by hot injection methods. ${ }^{28}$
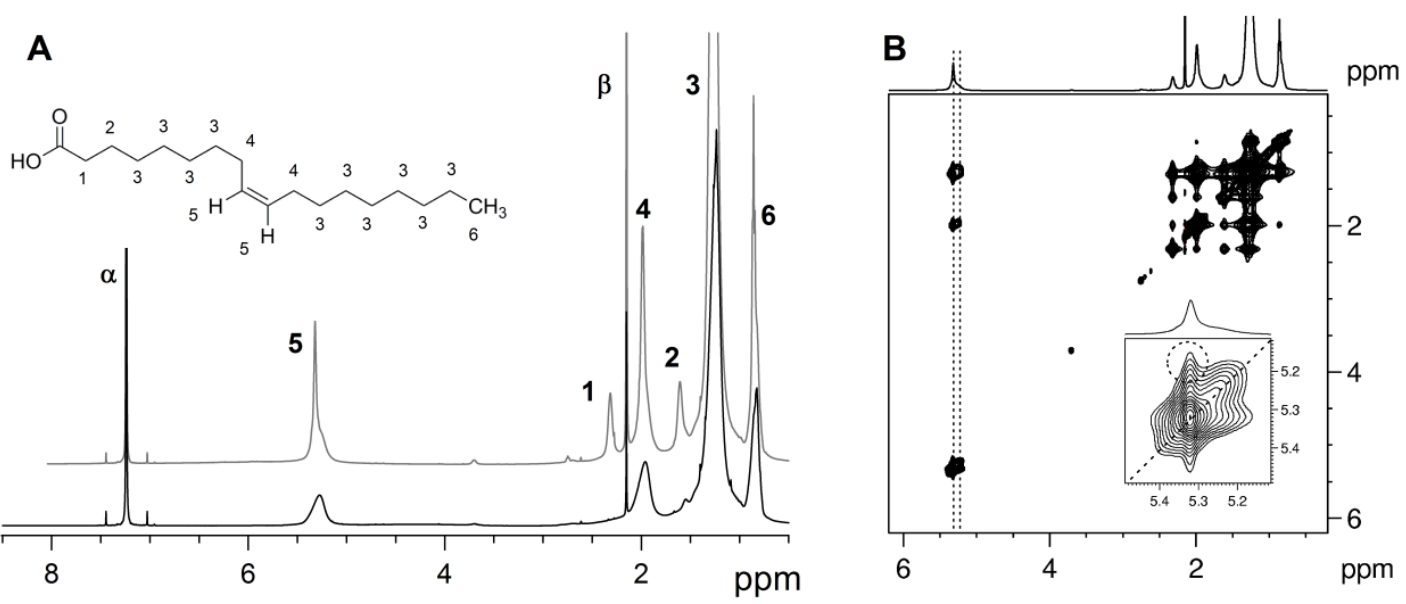

Figure 3. (A) ${ }^{1} \mathrm{H}$ NMR spectra of $\mathrm{HfO}_{2} \mathrm{NCs}$ in $\mathrm{CDCl}_{3}$, stabilized with $\mathrm{OAc}$ and OAm (black spectrum) after 5 purification steps and (grey spectrum) after subsequent addition of 1 equivalent of OAc with respect to bound oleic acid. Greek letters refer to resonances from the solvent and the nonsolvents used during purification. The protons of OAc are denoted by roman numbers. (B) 2D NOESY spectrum of the $\mathrm{HfO}_{2} \mathrm{NCs}$ stabilized with OAc after addition of 1 equivalent of excess OAc. The dotted lines are drawn to discern cross peaks of the broad and the sharp alkene resonances.

The surface composition of $\mathrm{PbS}$ and $\mathrm{CdSe} \mathrm{NCs}$ is already well understood and it was reported that a cation-rich core is charge balanced by X-type carboxylates. ${ }^{28-30}$ The question arises whether this is also the case for post-synthetically modified MONCs. A straightforward distinction between an adsorbed carboxylic acid or carboxylate is however not possible with NMR since the acidic protons, close to the surface, are indiscernible due to $\mathrm{T}_{2}$ relaxation.

For the exchange experiment, deuterated OAc $\left(d_{1}-\mathrm{OAc}\right)$ was prepared, see SI. The notation $d_{1}$-OAc signifies that only the acidic proton was replaced with deuterium. We added $d_{1}$-OAc ( 5 equivalents with respect to bound OAc) to a thoroughly purified, water-free suspension of oleic acid capped $\mathrm{HfO}_{2} \mathrm{NCs}$ in $\mathrm{CDCl}_{3}$. The detailed procedure can be found in the SI. The above mentioned exchange processes between bound OAc and excess $d_{1}$-OAc occur and the alleged surface protons - if any can be exchanged for deuterium, see eqs 1-2 in case of carboxylate or carboxylic acid ligands respectively. Note that this exchange is a purely stochastic event and merely causes a redistribution of proton and deuterium nuclei.

$$
\begin{gathered}
\mathrm{HfO}_{2} \cdot \mathrm{R}_{1} \mathrm{COO}+\mathrm{R}_{2} \mathrm{COOD} \rightleftharpoons \mathrm{HfO}_{2} \cdot \mathrm{R}_{2} \mathrm{COO}+\mathrm{R}_{1} \mathrm{COOD} \\
\mathrm{HfO}_{2} \cdot \mathrm{R}_{1} \mathrm{COOH}+\mathrm{R}_{2} \mathrm{COOD} \rightleftharpoons \mathrm{HfO}_{2} \cdot \mathrm{R}_{2} \mathrm{COOD}+\mathrm{R}_{1} \mathrm{COOH}
\end{gathered}
$$


The observed proton concentration (derived from the carboxylic acid signal at $12 \mathrm{ppm}$ ) relative to the total concentration of oleic acid molecules (from the alkene resonance) was plotted in Figure 4A, together with the theoretical predictions according to eqs 1-2. Even in the case of carboxylates on the surface (eq 1) the $[\mathrm{H}] /[\mathrm{OAc}]$ ratio is larger than naught because of the incomplete deuteration of $d_{1}$-OAc (92.34 \% deuterated). The corresponding ${ }^{1} \mathrm{H}$ NMR data and calculations are available in the SI. The experimental $[\mathrm{H}] /[\mathrm{OAc}]$ ratio matches the prediction based on eq 2 , which indicates that there are exactly as many protons near the surface as there are bound ligands.

Having the quantitative information of NMR, it would seem legitimate to regard these MONCs as stabilized by L-type carboxylic acids. However, the complete absence of a carboxylic acid absorption - expected at $1710 \mathrm{~cm}^{-1}$ - in the infrared spectrum of DDAc capped $\mathrm{HfO}_{2} \mathrm{NCs}$ (Figure 4B) conflicts with this hypothesis. In contrast, a carboxylate peak is detected at $1548 \mathrm{~cm}^{-1}$. In addition, around $3388 \mathrm{~cm}^{-1}$, a broad band is observed which is typical for hydrogen bonded O-H. ${ }^{37}$ The small peak at $3670 \mathrm{~cm}^{-1}$ is attributed to single hafnol moieties, Hf-O-H, similar to freestanding titanol. ${ }^{38}$ This again confirms (qualitatively) the presence of hydrogen atoms on the surface, however not bound to the carboxylate but directly to the nanocrystal. We conclude that the carboxylic acid is able to dissociate on the stoichiometric $\mathrm{HfO}_{2} \mathrm{NC}$ surface.


Figure 4. (A) The ratio of the proton concentration and the total oleic acid concentration for the addition of 5 equivalents of $d_{1}$-OAc. The experimental en theoretical values - dependent of the assumption of carboxylate or carboxylic acid on the surface - are given. (B) ATR-FTIR spectrum of dried $\mathrm{HfO}_{2} \mathrm{NCs}$ capped with dodecanoic acid.

3.5 Is OAm imperative for stabilization? The surface before and after modification utterly clarified, the question remains why OAm is indispensable. The experiment in section 3.3 points out that OAm is not strongly bound to the surface of the NCs. Even more, OAm seems not even relevant to the stabilization process as the NCs are perfectly stable after OAm being removed. To determine unambiguously the influence of the amine, a combination of 10-undecenoic acid and OAm was used since both have different characteristic resonances outside the aliphatic region. Figure 5A displays the ${ }^{1} \mathrm{H}$ NMR spectra of $\mathrm{HfO}_{2} \mathrm{NCs}$ with 10-undecenoic acid in $\mathrm{CDCl}_{3}$, before 
and after addition of OAm. Note the presence of diethyl ether $(\beta)$ which was used to wash the NCs prior to suspension in chloroform. Before any OAm was added, only sharp resonances of 10undecenoic acid were observed. This suggests that the acid does not interact with the NC surface, a conclusion confirmed by the 2D NOESY spectrum (Figure 5B) which only features small, positive nOe crosspeaks and zero quantum coherences typical of free ligands. The particles precipitate quickly, as could be expected for an unstabilized system.
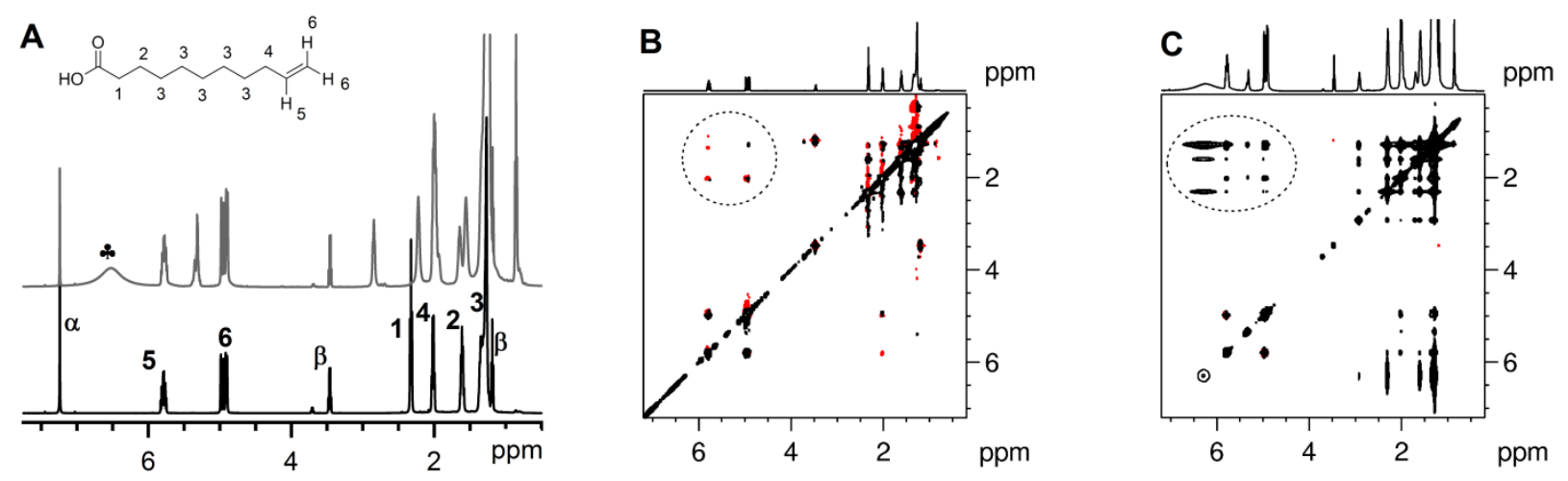

Figure 5. (A) ${ }^{1} \mathrm{H}$ proton spectra of $\mathrm{HfO}_{2} \mathrm{NCs}_{\text {in }} \mathrm{CDCl}_{3}$ (black spectrum) with 10-undecenoic acid and (grey spectrum) with 10-undecenoic acid and OAm, concentration: $28 \mathrm{mg} \mathrm{HfO} 2 / \mathrm{mL}$. Greek letters refer to resonances from the solvent and the nonsolvents used during purification. The broad clover resonance is attributed to an ammonium compound (see section 3.5 (B) 2D NOESY of $\mathrm{HfO}_{2} \mathrm{NCs}$ in $\mathrm{CDCl}_{3}$ with 10-undecenoic acid (C) 2D NOESY of $\mathrm{HfO}_{2} \mathrm{NCs}$ in $\mathrm{CDCl}_{3}$ with 10-undecenoic acid and OAm.

Upon addition of only $13 \mathrm{mM}$ of OAm (i.e., $10 \mathrm{~mol} \%$ with respect to hafnium) - an amount insufficient to fully de-aggregate and stabilize the turbid suspension yet enough to avoid precipitation - the resonances of OAm appear in the ${ }^{1} \mathrm{H}$ NMR spectrum and a slight broadening of all signals (except the solvent) is observed (see Figure 5A). Moreover, both 10-undecenoic acid and OAm now feature negative nOe's, indicating interaction with the NC surface (see Figure 5C). In line with this conclusion, DOSY yields two diffusion coefficients for 10-undecenoic acid, corresponding to free $\left(842 \pm 2 \mu \mathrm{m}^{2} / \mathrm{s}\right)$ and bound $\left(96 \pm 6 \mu \mathrm{m}^{2} / \mathrm{s}\right)$ moieties, respectively. The signals of OAm on the other hand exhibit a mono exponential decay in DOSY, with a diffusion coefficient of $307 \pm 5 \mu \mathrm{m}^{2} / \mathrm{s}$ in between that of free OAm $\left(864 \pm 1 \mu \mathrm{m}^{2} / \mathrm{s}\right)$ and bound OAm. As observed previously with $\mathrm{CdSe}, \mathrm{CdTe}, \mathrm{PbS}$ and $\mathrm{ZnO} \mathrm{NCs}$, this indicates that $\mathrm{OAm}$ is in fast exchange between a bound and a free state, ${ }^{39}$ a conclusion in line with the observation that OAm is readily removed by successive purification. However, the most far-reaching result is that the carboxylic acid will only bind to the $\mathrm{NC}$ surface if OAm is present, i.e., although absent in the eventual ligand shell, the amine is essential to the ligand exchange.

Returning to section 3.3, XRF measurements (SI) indicate that traces of chlorine are still present in sample 1 (purified only once) but not in sample 2 (purified 5 times). Together with the removal of 
chlorine the broad resonance - indicated by the clover symbol \& in Figure 2 - disappears upon successive purification. The clover resonance might be assigned to an ammonium compound and this suggests that the purification removes oleylammonium chloride together with the excess of OAm. Further indication that the clover resonance is OAm related is given by Figure $5 \mathrm{~A}$ where again the broad feature is observed upon addition of OAm, although with a somewhat different chemical shift. This is not uncommon since the ammonium proton is easily exchanged, which makes its position dependent on the amount of amine present in the sample and its chemical environment.

Provided that OAm merely serves as a base, the use of other bases - together with carboxylic acids - should equally lead to the successful surface modification of $\mathrm{HfO}_{2}$ or $\mathrm{ZrO}_{2} \mathrm{NCs}$. In Figure 6, DLS measurements are shown of three samples modified with three different bases. In case of OAm and triethylamine, only a stoichiometric amount was needed to ensure immediate stabilization, confirming that only a base is required. The small difference between triethylamine and OAm despite the similar $\mathrm{pK}_{\mathrm{a}}$ (in water) is attributed to steric hindrance of the tertiary amine. However, in case of pyridine, significant aggregation persists, even when used in large excess. As discussed below, this is attributed to insufficient basicity.

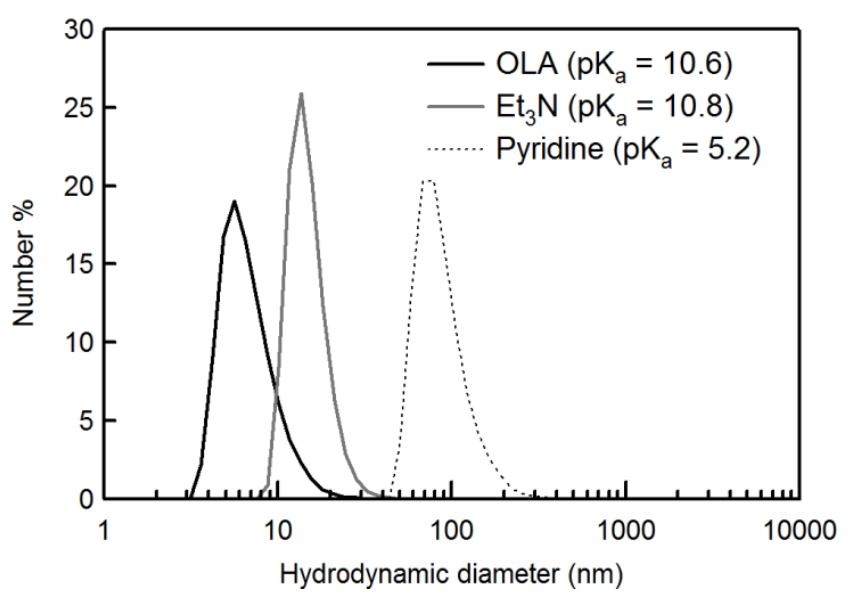

Figure 6. DLS measurements of $\mathrm{HfO}_{2} \mathrm{NCs}_{\text {in }} \mathrm{CHCl}_{3}(15 \mathrm{mg} \mathrm{HfO} / 2 \mathrm{~mL})$ prepared by post modification with DDAc (50 mol\% with respect to $\mathrm{Hf}$ ) and either oleylamine (50 mol\% with respect to $\mathrm{Hf}$ ), triethylamine ( $50 \mathrm{~mol} \%$ with respect to $\mathrm{Hf}$ ) or pyridine ( $270 \mathrm{~mol} \%$ with respect to $\mathrm{Hf}$ ).

\section{Discussion}

In line with previous studies on $\mathrm{CdSe},{ }^{28,40} \mathrm{PbS}^{41,42}$ and $\mathrm{InP}^{43} \mathrm{NCs}$, we have demonstrated that carboxylic acids bind as carboxylates to $\mathrm{HfO}_{2}$ and $\mathrm{ZrO}_{2}$ NCs. Using IR spectroscopy, similar conclusions were arrived at for $\mathrm{Fe}_{2} \mathrm{O}_{3},{ }^{17} \mathrm{MFe}_{2} \mathrm{O}_{4}(\mathrm{M}=\mathrm{Fe}, \mathrm{Mn}$, and $\mathrm{Co}),{ }^{44} \mathrm{ZnO}^{45}$ and $\mathrm{ZrO}_{2}{ }^{46} \mathrm{NCs}$, yet the combination with NMR spectroscopy enables us to complement this finding with the observation that in the case of $\mathrm{HfO}_{2}$ and $\mathrm{ZrO}_{2}$, the negative charge on the carboxylate moieties is 
balanced by surface adsorption of protons rather than by excess metal cations. Moreover, carboxylic acids will only bind as carboxylates on the as-synthesized NCs provided that a sufficient amount of oleylamine is supplied, which removes hydrogen chloride, initially present at the surface. This provides us with the possibility to use cheap chloride precursors without excluding applications where chloride is detrimental.

This set of results can be rationalized by considering the occurrence of acid-base reactions at the MONC surface. Before surface modification, in aqueous (or other polar) media, protons adsorb on the surface of the MONCs, thereby charging the surface (eq 3).

$$
\mathrm{MO}_{2}(\mathrm{~s})+\mathrm{HCl}(\mathrm{aq}) \rightarrow \mathrm{MO}_{2} \cdot \mathrm{H}^{+}(\mathrm{aq})+\mathrm{Cl}^{-}(\mathrm{aq})
$$

Since the system in chloroform has to be charge neutral, the $\mathrm{MO}_{2} \mathrm{NC}$ precipitates are more likely described as $\mathrm{MO}_{2} \cdot \mathrm{H}^{+} \mathrm{Cl}^{-}(\mathrm{s})$, i.e., as NCs having Brønsted acids adsorbed at their surface. Importantly, this notation does not imply that the proton and the chloride ion are bound to one another. They may well occupy different adsorption sites on the NC. During the surface modification the chloride anions are replaced by carboxylates resulting in a stabilized colloidal suspension of aggregate-free MONCs (eq 4).

$$
\begin{gathered}
\mathrm{MO}_{2} \cdot \mathrm{H}^{+} \mathrm{Cl}^{-}+\mathrm{RCOOH} \rightleftharpoons \mathrm{MO}_{2} \cdot \mathrm{H}^{+} \mathrm{RCOO}^{-}+\mathrm{HCl} \\
\mathrm{MO}_{2} \cdot \mathrm{H}^{+} \mathrm{Cl}^{-}+\mathrm{OAm} \rightleftharpoons \mathrm{MO}_{2}+\mathrm{OAmH}^{+} \mathrm{Cl}^{-} \\
\mathrm{RCOOH}+\mathrm{OAm} \rightleftharpoons \mathrm{RCOO}^{-}+\mathrm{OAmH}^{+} \\
\mathrm{MO}_{2}+\mathrm{RCOOH} \rightleftharpoons \mathrm{MO}_{2} \cdot \mathrm{H}^{+} \mathrm{RCOO}^{-} \\
\mathrm{MO}_{2} \cdot \mathrm{H}^{+} \mathrm{Cl}^{-}+\mathrm{RCOO}
\end{gathered}
$$

This reaction is however unfavorable in chloroform because of the high solubility of the carboxylic acid, the low solubility of $\mathrm{HCl}$ and the difference in $\mathrm{pK}_{\mathrm{a}}$. The role of OAm is to lower the activity of either the adsorbed protons (eq 5) or the proton of the carboxylic acid (eq 6). In the first case the acid can subsequently dissociate on the bare NC surface (eq 7) and in the latter case the carboxylate can exchange for the chloride (eq 8) but the net effect remains the same, i.e., the equilibrium of eq 4 is displaced to the right by the formation of an ammonium chloride salt. In apolar solutions, the salt remains in the ligand shell, until it leaches out during the purification with polar solvents. A model for the overall reaction during the surface modification is presented in scheme 1, which indicates the changes in surface chemistry and stresses to role of the amine as a base. In general any base would comply but in practice the options are limited due to solubility and basicity, e.g., pyridine is too weak a base $\left(\mathrm{pK}_{\mathrm{a}}=5.2\right)$, which means that it cannot fully deprotonate the carboxylic acid. 


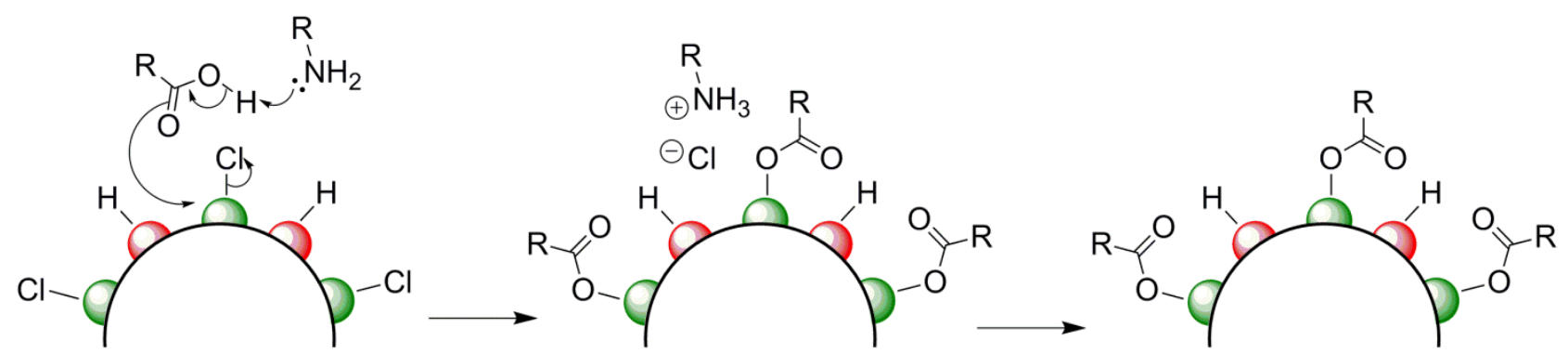

Scheme 1. In the first step the surface modification mechanism is displayed. The amine captures a proton of the carboxylic acid. Subsequently the carboxylate exchanges for the chloride. The chloride is paired with the ammonium compound as a salt and remains in the ligand shell. In the second step the NC are purified (precipitated and redispersed) with polar solvents and the salt leaches out of the ligand shell. The final result is a dissociated carboxylic acid on the $\mathrm{HfO}_{2}$ or $\mathrm{ZrO}_{2} \mathrm{NC}$ surface.

Scheme 1 shows that MONCs charge stabilized in polar media by the (dissociative) adsorption of a Brønsted acid can be transferred to apolar media by acid exchange. It indicates that the factors governing this exchange reactions are the $\mathrm{pKa}$ of the two acids, the binding affinity of the conjugate bases to the MONC surface and the solubility of the reagents and products involved. Working on similar functionalization experiments in the case of indium tin oxide NCs, Grote et al. deducted from TGA experiments that benzyl alcohol $(\mathrm{BnOH})$ is present on the surface of as-synthesized ITO NCs and the authors concluded that amines do not bind sufficiently strong to replace the -OBn groups. In regard of the above surface chemistry model, we can conclude that an important parameter preventing this exchange will be acidity. To remove the -OBn moiety, it would need to be protonated $\left(\mathrm{pK}_{\mathrm{a}}\right.$ of $\left.\mathrm{BnOH} \approx 15\right)$ but the amines are too weak an acid $\left(\mathrm{pK}_{\mathrm{a}} \approx 35\right)$ to render this exchange possible. Hence the use of our model to understand the surface chemistry of MONCs and judiciously modify their surface.

The observation that dissociated carboxylic acids are present on the MONC surface in nonpolar media - see the end result of scheme 1 for visual representation - is unprecedented. Metal sulfide, selenide and telluride nanocrystals for example were always found to be stabilized by carboxylate or phosphonate moieties that are charge balanced by a metal cation excess, a combination that can be described as the salt of the metal and the conjugated base of a carboxylic or phosphonic acid. Although scheme 1 specifically represents the surface modification of $\mathrm{HfO}_{2}$ and $\mathrm{ZrO}_{2}$ synthesized via benzyl alcohol and metal chlorides, the underlying surface chemistry model of stabilization by a dissociated Brønsted acid may apply to MONCs in general. Indeed, since the ability of a NC surface to adsorb protons will depend on the Brønsted basicity of the chalcogen, dissociative adsorption of acids is unlikely for metal chalcogenide NCs of the heavier chalcogens ( $\mathrm{S}, \mathrm{Se}, \mathrm{Te})$ yet it can be a common characteristic of MONCs. The observation that at least for $\mathrm{HfO}_{2}$ and $\mathrm{ZrO}_{2}$, the $\mathrm{NC}$ surface provides a medium where acid base reactions can proceed in nonpolar solvents extends considerably the possibility for ligand exchange reactions with these MONCs. Instead of only exchanging the anionic species - as is common practice with metal sulfide, selenide or telluride 
$\mathrm{NCs}^{23,24,47}$ - the MONC surface can be modified by exchanging the proton for other cations. Moreover, Brønsted acids should now be seen as offering a pair of X-type ligands - the proton and the conjugated base - rather than a single X-type ligand, which implies that the need for proton transfer will no longer restrict ligand exchange processes. As a result, adsorption/desorption equilibria can exist between a dissolved Brønsted acid and the adsorbed conjugate base/proton pair and the direct exchange of this conjugate base/proton pair for L-type ligands may be possible.

\section{Conclusion}

We have elucidated the surface modification mechanism of $\mathrm{HfO}_{2}$ and $\mathrm{ZrO}_{2} \mathrm{NCs}$, synthesized via surfactant-free nonaqueous methods from the respective metal chlorides and benzyl alcohol. We showed that the carboxylic acid is unable to replace the initially present hydrogen chloride without the presence of a suitable base such as oleylamine. Furthermore, oleylamine is only weakly entangled in the ligand shell and can be removed by simple purification together with undesired traces of chloride, leaving a clean surface suitable for various applications. After surface functionalization of the metal oxide NCs, the carboxylic acids feature the same dynamical behavior in solution as metal chalcogenide NCs stabilized with carboxylates. However, we established the crucial difference that in case of metal oxides the negative charge of the carboxylate is not balanced by excess of cations but by protons which are adsorbed on different adsorption sites on the surface than the carboxylates. We conclude that carboxylic acids can dissociate on the surface of the metal oxide NCs which is an unprecedented and fascinating result that opens new possibilities for the manipulation of metal oxide NCs in general.

\section{Associated Content}

\section{Supporting information}

Comments on the experimental procedure, characterization of $\mathrm{ZrO}_{2} \mathrm{NC}$ suspensions, ROESY spectra of $\mathrm{HfO}_{2} \mathrm{NCs}$ with oleic acid, details on the $d_{1}$-OAc titration and XRF results. This information is available free of charge via the internet at http://pubs.acs.org/

\section{Acknowlegdements}

We would like to express our gratitude to dr. Jonas Feys for XRD measurements. Prof. dr. Johan Van der Eycken is acknowledged for the use of the ATR-FTIR device. J.D.R acknowledges the FWO-Vlaanderen for funding, Z.H and J.C.M acknowledge SIM for funding (SIM-SIBO SOPPOM). Z.H acknowledges BelSPo (IAP 7.35, photonics@be), the FWO-Vlaanderen (research project G.0760.12) and UGent for funding (GOA and BOF project). 


\section{References}

(1) Lee, N.; Cho, H. R.; Oh, M. H.; Lee, S. H.; Kim, K.; Kim, B. H.; Shin, K.; Ahn, T.-Y.; Choi, J. W.; Kim, Y.-W.; Choi, S. H.; Hyeon, T. J. Am. Chem. Soc. 2012, 134, 10309.

(2) Zou, Z. G.; Ye, J. H.; Sayama, K.; Arakawa, H. Nature 2001, 414, 625.

(3) Yamada, Y.; Tsung, C. K.; Huang, W.; Huo, Z. Y.; Habas, S. E.; Soejima, T.; Aliaga, C. E.; Somorjai, G. A.; Yang, P. D. Nat. Chem. 2011, 3, 372.

(4) Comini, E.; Faglia, G.; Sberveglieri, G.; Pan, Z. W.; Wang, Z. L. Appl. Phys. Lett. 2002, 81, 1869.

(5) Lee, J.; Zhang, S.; Sun, S. H. Chem. Mat. 2013, 25, 1293.

(6) Mor, G. K.; Varghese, O. K.; Paulose, M.; Shankar, K.; Grimes, C. A. Sol. Energy Mater. Sol. Cells 2006, 90, 2011.

(7) O'Dwyer, C.; Szachowicz, M.; Visimberga, G.; Lavayen, V.; Newcomb, S. B.; Torres, C. M. S. Nat. Nanotechnol. 2009, 4, 239.

(8) Poizot, P.; Laruelle, S.; Grugeon, S.; Dupont, L.; Tarascon, J. M. Nature 2000, 407, 496.

(9) Sun, Y. K.; Chen, Z. H.; Noh, H. J.; Lee, D. J.; Jung, H. G.; Ren, Y.; Wang, S.; Yoon, C. S.; Myung, S. T.; Amine, K. Nat. Mater. 2012, 11, 942.

(10) Wang, Z. L. Mater. Sci. Eng. R-Rep. 2009, 64, 33.

(11) Matijevic, E. Chem. Mat. 1993, 5, 412.

(12) Matijevic, E. Accounts Chem. Res. 1981, 14, 22.

(13) Park, J.; Joo, J.; Kwon, S. G.; Jang, Y.; Hyeon, T. Angew. Chem.-Int. Edit. 2007, 46, 4630.

(14) Jana, N. R.; Chen, Y. F.; Peng, X. G. Chem. Mat. 2004, 16, 3931.

(15) Jun, Y. W.; Choi, J. S.; Cheon, J. Angew. Chem.-Int. Edit. 2006, 45, 3414.

(16) Ito, D.; Yokoyama, S.; Zaikova, T.; Masuko, K.; Hutchison, J. E. ACS Nano 2014, 8, 64.

(17) Willis, A. L.; Turro, N. J.; O'Brien, S. Chem. Mat. 2005, 17, 5970.

(18) Wang, F.; Tang, R.; Kao, J. L. F.; Dingman, S. D.; Buhro, W. E. J. Am. Chem. Soc. 2009, 131, 4983.

(19) Wang, F. D.; Tang, R.; Buhro, W. E. Nano Lett. 2008, 8, 3521.

(20) Pinna, N.; Niederberger, M. Angew. Chem.-Int. Edit. 2008, 47, 5292.

(21) De Roo, J.; De Keukeleere, K.; Feys, J.; Lommens, P.; Hens, Z.; Van Driessche, I. J. Nanopart. Res. 2013, 15, 1778.

(22) Bilecka, I.; Djerdj, I.; Niederberger, M. Chem. Commun. 2008, 886.

(23) Liu, W. Y.; Lee, J. S.; Talapin, D. V. J. Am. Chem. Soc. 2013, 135, 1349.

(24) Nag, A.; Kovalenko, M. V.; Lee, J. S.; Liu, W. Y.; Spokoyny, B.; Talapin, D. V. J. Am. Chem. Soc. 2011, 133, 10612.

(25) Grote, C.; Cheema, T. A.; Garnweitner, G. Langmuir 2012, 28, 14395.

(26) Garnweitner, G.; Goldenberg, L. M.; Sakhno, O. V.; Antonietti, M.; Niederberger, M.; Stumpe, J. Small 2007, 3, 1626.

(27) Pinna, N.; Grancharov, S.; Beato, P.; Bonville, P.; Antonietti, M.; Niederberger, M. Chem. Mat. 2005, 17, 3044.

(28) Fritzinger, B.; Capek, R. K.; Lambert, K.; Martins, J. C.; Hens, Z. J. Am. Chem. Soc. 2010, 132, 10195. 15081.

(29) Moreels, I.; Fritzinger, B.; Martins, J. C.; Hens, Z. J. Am. Chem. Soc. 2008, 130,

(30) Anderson, N. C.; Owen, J. S. Chem. Mat. 2013, 25, 69. 12279.

(31) Owen, J. S.; Park, J.; Trudeau, P. E.; Alivisatos, A. P. J. Am. Chem. Soc. 2008, 130,

(32) Desvaux, H.; Goldman, M. J. Magn. Reson. Ser. B 1996, 110, 198. 
(33) Connell, M. A.; Bowyer, P. J.; Bone, P. A.; Davis, A. L.; Swanson, A. G.; Nilsson, M.; Morris, G. A. J. Magn. Reson. 2009, 198, 121.

(34) Sinnaeve, D. Concepts Magn. Reson. Part A 2012, 40A, 39.

(35) Hassinen, A.; Moreels, I.; De Nolf, K.; Smet, P. F.; Martins, J. C.; Hens, Z. J. Am. Chem. Soc. 2012, 134, 20705.

(36) Hens, Z.; Martins, J. C. Chem. Mat. 2013, 25, 1211.

(37) Lao, S. X.; Martin, R. M.; Chang, J. P. J. Vac. Sci. Technol. A 2005, 23, 488.

(38) Yates, D. J. C. J. Phys. Chem. 1960, 65, 746.

(39) Fritzinger, B.; Moreels, I.; Lommens, P.; Koole, R.; Hens, Z.; Martins, J. C. J. Am. Chem. Soc. 2009, 131, 3024.

(40) Anderson, N. C.; Hendricks, M. P.; Choi, J. J.; Owen, J. S. J. Am. Chem. Soc. 2013, $135,18536$.

(41) Moreels, I.; Justo, Y.; De Geyter, B.; Haustraete, K.; Martins, J. C.; Hens, Z. ACS Nano 2011, 5, 2004.

(42) Cass, L. C.; Malicki, M.; Weiss, E. A. Anal. Chem. 2013, 85, 6974.

(43) Cros-Gagneux, A.; Delpech, F.; Nayral, C.; Cornejo, A.; Coppel, Y.; Chaudret, B. J. Am. Chem. Soc. 2010, 132, 18147.

(44) Cabrera, L. I.; Somoza, A.; Marco, J. F.; Serna, C. J.; Morales, M. P. J. Nanopart. Res. 2012, 14.

(45) Damm, H.; Kelchtermans, A.; Bertha, A.; Van den Broeck, F.; Elen, K.; Martins, J. C.; Carleer, R.; D'Haen, J.; De Dobbelaere, C.; Hadermann, J.; Hardy, A.; Van Bael, M. K. RSC Adv. 2013, 3, 23745.

(46) Taguchi, M.; Takami, S.; Adschiri, T.; Nakane, T.; Sato, K.; Naka, T. Crystengcomm 2012, 14, 2132.

(47) Dewi, M. R.; Skinner, W. M.; Nann, T. Australian Journal of Chemistry 2014, 67, 663. 


\section{ToC Graphic}

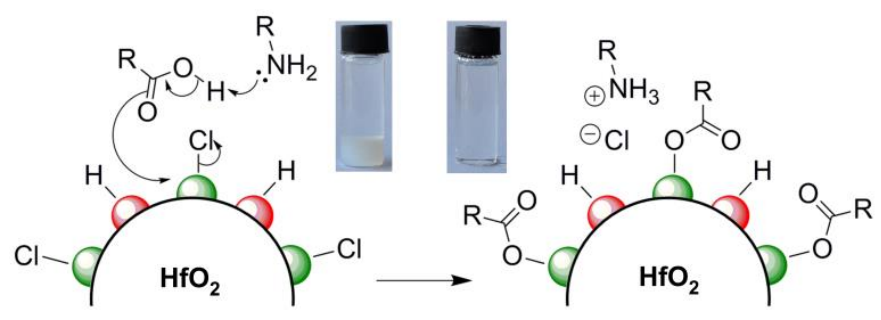

